\title{
Incorporation of Iron Oxide Nanoparticles and Quantum Dots into Silica Microspheres
}

\section{Citation}

Insin, Numpon, Joseph B. Tracy, Hakho Lee, John P. Zimmer, Robert M. Westervelt, and Moungi G. Bawendi. 2008. Incorporation of iron oxide nanoparticles and quantum dots into silica microspheres. ACS Nano 2(2): 197-202.

\section{Published Version}

doi: $10.1021 / \mathrm{nn} 700344 \mathrm{x}$

\section{Permanent link}

http://nrs.harvard.edu/urn-3:HUL.InstRepos:4728507

\section{Terms of Use}

This article was downloaded from Harvard University's DASH repository, and is made available under the terms and conditions applicable to Open Access Policy Articles, as set forth at http:// nrs.harvard.edu/urn-3:HUL.InstRepos:dash.current.terms-of-use\#OAP

\section{Share Your Story}

The Harvard community has made this article openly available.

Please share how this access benefits you. Submit a story.

\section{Accessibility}


Incorporation of Iron Oxide Nanoparticles and Quantum Dots into Silica Microspheres

\begin{tabular}{|r|l|}
\hline Journal: & ACS Nano \\
\hline Manuscript ID: & nn-2007-00344x \\
\hline Manuscript Type: & Article \\
\hline Complete List of Authors: & $\begin{array}{l}\text { Insin, Numpon; MIT, Chemistry } \\
\text { Tracy, Joseph; North Carolina State Universit, Materials Science and } \\
\text { Engineering } \\
\text { Lee, Hakho; Masachusetts General Hospital, Center for Molecular } \\
\text { Imaging Research } \\
\text { Zimmer, John; MIT, Chemistry } \\
\text { Westervelt, Robert; Harvard University, Dept. of Engineering \& } \\
\text { Applied Science } \\
\text { Bawendi, Moungi; MIT, Chemistry }\end{array}$ \\
\hline
\end{tabular}

\section{(5) ScholarONE \\ Manuscript Central}




\title{
Incorporation of Iron Oxide Nanoparticles and Quantum Dots into Silica Microspheres
}

\author{
Numpon Insin, ${ }^{\dagger}$ Joseph B. Tracy, ${ }^{\dagger}$ Hakho Lee, ${ }^{\dagger \dagger}$ John P. Zimmer, ${ }^{\dagger}$ \\ Robert M. Westervelt, ${ }^{\dagger \dagger}$ and Moungi G. Bawendi ${ }^{\dagger, *}$ \\ ${ }^{\dagger}$ Department of Chemistry, Massachusetts Institute of Technology, Cambridge, MA \\ 02139 \\ ${ }^{\dagger}$ Division of Engineering and Applied Sciences and Department of Physics, Harvard \\ University, Cambridge, MA 02138 \\ *Address correspondence to $\underline{\mathrm{mgb} @ \text { mit.edu }}$
}

\begin{abstract}
We describe the synthesis of magnetic and fluorescent silica microspheres, fabricated by incorporation of maghemite $\left(\gamma-\mathrm{Fe}_{2} \mathrm{O}_{3}\right)$ nanoparticles (MPs) and $\mathrm{CdSe} / \mathrm{CdZnS}$ core shell quantum dots (QDs) into a silica shell around pre-formed silica microspheres. The resultant $\sim 500 \mathrm{~nm}$ microspheres have narrow size distribution and show uniform incorporation of QDs and MPs into the shell. We demonstrated manipulation of these microspheres using an external magnetic field with real-time monitoring under a fluorescent light microscope.
\end{abstract}


Magnetic silica microspheres are of great interest for biomedical ${ }^{1}$ and environmental research applications. Magnetic microspheres of various types have been used for bioseparation, drug targeting, cell isolation, enzyme immobilization, protein purification, and wastewater treatment. ${ }^{2-4}$ High stability and biocompatibility make silica microspheres preferred over other types. ${ }^{2,5,6}$ In addition, silica microspheres can be easily modified with a wide range of functional groups. ${ }^{6}$ As a result, magnetic silica microspheres have been widely studied by many techniques, such as layer-by-layer self-assembly, ${ }^{2}$ the Stöber process, ${ }^{7}$ ferrite plating, ${ }^{8}$ aerosol pyrolysis, ${ }^{9}$ and sonochemical deposition. ${ }^{10}$

For the preparation of magnetic silica microspheres, incorporation of ferromagnetic or ferromagnetic nanopartices is desirable. These magnetic nanoparticles (MPs) range in size from a few nanometers to tens of nanometers. Sufficiently small MPs in this size regime exist as single-domain magnets, ${ }^{11}$ in which each MP has a constant magnetic moment, which can be reoriented in an applied field. When small MPs are heated from low temperature, thermal energy begins to perturb their orientational stability, which is known as superparamagnetism. Superparamagnetic MPs can be oriented in an applied field, but they have no net magnetization in zero field. The onset of superparamagnetism is gradual, and the blocking temperature $\left(T_{B}\right)$, which is proportional to the MP volume, demarcates the transition to superparamagnetism. Small superparamagnetic MPs with low $T_{B}$ are much less susceptible to aggregation than larger ones. MPs have been utilized in various biomedical applications, such as magnetic separation, drug delivery, magnetic resonance imaging (MRI), and hyperthermia treatment. ${ }^{1,}{ }^{12}$ Among MPs, maghemite or $\gamma-\mathrm{Fe}_{2} \mathrm{O}_{3}$ nanoparticles are some of the most promising for in vivo applications because of the known biocompatibility of $\gamma$ $\mathrm{Fe}_{2} \mathrm{O}_{3}{ }^{12}$

Incorporating MPs along with chromophores enables additional applications. Such microspheres can be moved with an external magnetic field while monitoring their motion through fluorescence in real time. ${ }^{13,14}$ Fluorescent and magnetic microspheres have been fabricated by combining magnetic nanoparticles with organic dyes or lanthanide metal complexes. ${ }^{13,14}$ Advantages of using QD as fluorophores are their continuous adsorption spectra, narrow emission bandwidths, and large two photon absorption cross-section. ${ }^{15-17}$

Due to the advantages of a system which combines a substantial magnetic moment and luminescence, composite silica microspheres containing both MPs and QDs have attracted great interest. ${ }^{5,6,18,19}$ Previous fabrication methods included the use of MPs and QDs as cores followed by the growth of a silica shell, ${ }^{6,18}$ and the inverse suspension method. ${ }^{5}$ 
However, most samples prepared using these methods were polydisperse. ${ }^{5,18}$ Moreover, the numbers of MPs and QDs in each particle within the same sample were not uniform. 5, 6, 18 More recently, magnetic and fluorescent silica microspheres were prepared by using silicacoated MPs as cores, followed by layer-by-layer (LbL) assembly of polyelectrolyte and QDs onto the cores' surfaces, which were then coated with a final silica shell. ${ }^{19}$ However, the sizedispersity of the particles was not characterized, and the MP content in each MS was limited and uncontrolled. In addition, the technique of polyelectrolyte-assisted QD assembly limited the number of QDs adsorbed onto the cores' surfaces to a monolayer. This technique also used QDs with negatively-charged surfaces prepared with an aqueous method, ${ }^{20}$ which was known to yield QDs with lower crystallinity, monodispersity, and fluorescence efficiency than QDs prepared in nonaqueous coordinating solvents using the "hot-injection" technique. $^{20,21}$

Here we report the synthesis of monodisperse silica microspheres with MPs and QDs both uniformly incorporated, and demonstrate their practical bifunctionality. Potential applications of these microspheres include monitoring drug delivery and the combination of deep tissue MRI imaging with high resolution confocal laser scanning microscopy.

Our approach is based on our previous work, in which QDs were incorporated into silica shells grown on pre-made silica microsphere cores through a sol-gel process. ${ }^{15}$ This fabrication method is advantageous because it maintains the monodisperse MS size and QD optical properties, and the nanoparticles are incorporated uniformly into the MS shell. We have modified the shell of the microspheres to include not only QDs, but MPs as well. Preparing the nanoparticle stock solution is the most important step in the process. QDs and MPs must be highly soluble in ethanol and also possess accessible alkoxysilane groups, which polymerize with tetraethoxysilane (TEOS) to form the shell.

Scanning electron microscopy (SEM) and transmission electron microscopy (TEM) show that the morphology of the core microspheres (Figure 1b,c) did not change upon the addition of QD- and MP-doped shells (Figure 1e,f), but rougher surfaces were observed. Size distributions (Figure 1d,g) measured from TEM images indicated that the incorporation process did not significantly affect the size dispersity of the microspheres. Moreover, the increase in average size of the overcoated microspheres confirmed that the core/shell structure was formed. 


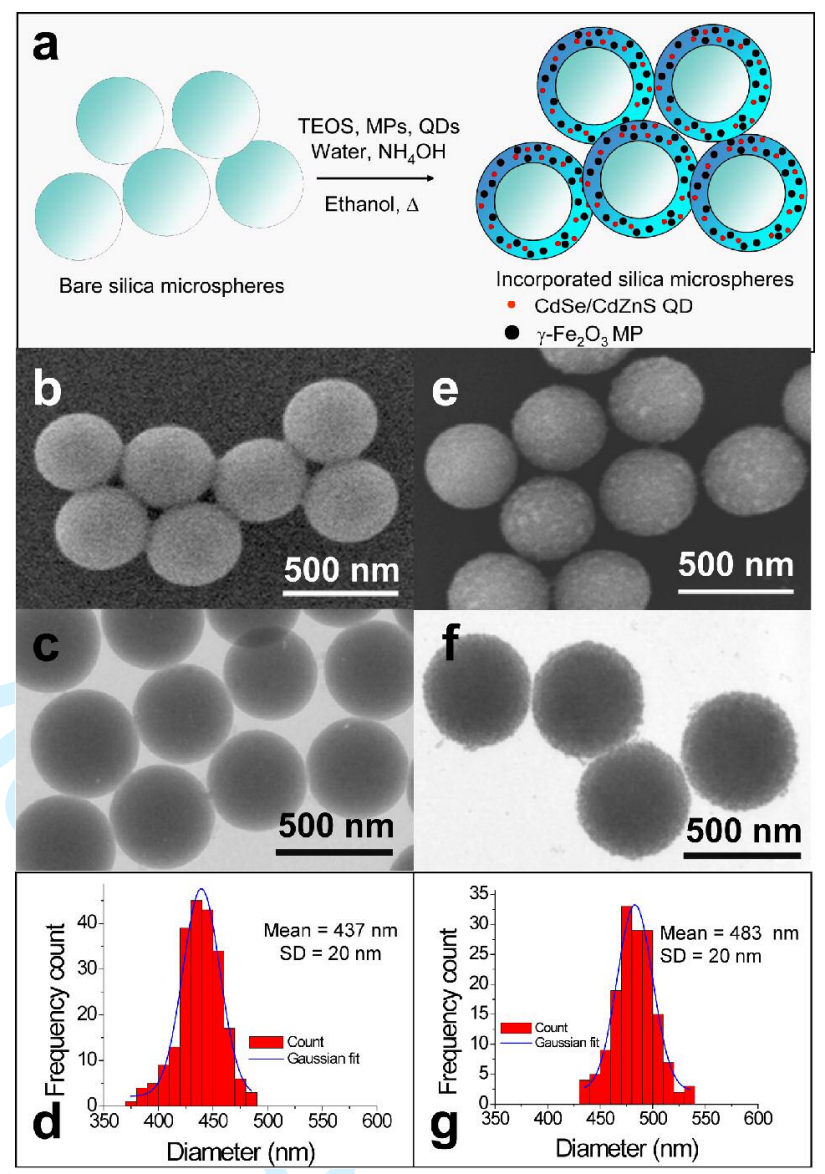

Figure 1. (a) Reaction scheme for the incorporation of silica microspheres (b-d) $500 \mathrm{~nm}$ silica microsphere (Polysciences, $500 \pm 70 \mathrm{~nm}$ ) before incorporation (b) SEM image (c) TEM image (d) size distribution analysis (e-g) $500 \mathrm{~nm}$ silica microspheres after incorporation of QDs and MPs (7-nm MP, 12000 MPs per microsphere), (e) SEM image (f) TEM image (g) size distribution analysis.

As noted above, the crucial step for incorporating MPs into silica microspheres was the preparation of MP stock solution in ethanol. The MPs' native surfactant, oleic acid or stearic acid, was displaced by 5-amino-1-pentanol (AP) and 3-aminopropyltrimethoxysilane (APS) in order for the MPs to become ethanol-soluble and polymerizable with TEOS, respectively. Addition of a small amount of 12-hydroxydodecanoic acid (HDDA) helped increase the number of MPs incorporated into the microspheres. For instance, in the incorporation of 7-nm MPs into 500-nm microspheres, the MP content was as high as 13000 MPs per microsphere when HDDA was added to the MP solution. When no HDDA was added, however, the highest MP content achievable before the aggregation of MPs outside the microspheres was observed was four times less. The improved incorporation was most likely due to the increased solubility of MPs in ethanol, which could reduce the rate of selfcondensation of APS, which was probably the reason for MP aggregation outside microspheres. 
Fluorescence microscopy showed that the microspheres were suitably bright for imaging applications. In addition, every microsphere exhibited QD fluorescence of similar intensities, implying that the QDs were incorporated and distributed uniformly as seen in Figure 2a,b.

The distribution of iron was observed using scanning transmission electron microscopy (STEM) equipped with an energy-dispersive X-ray analyzer, as shown in Figure 2c. Uniform distribution among many microspheres was observed. Moreover, this plot of iron (red spots) and silicon atoms (white spots), identified the shell as the area of dense distribution of iron atoms. This observation, combined with data from a line scanning across a single MS (see Supporting Information), indicated a shell thickness of $55 \pm 10 \mathrm{~nm}$.

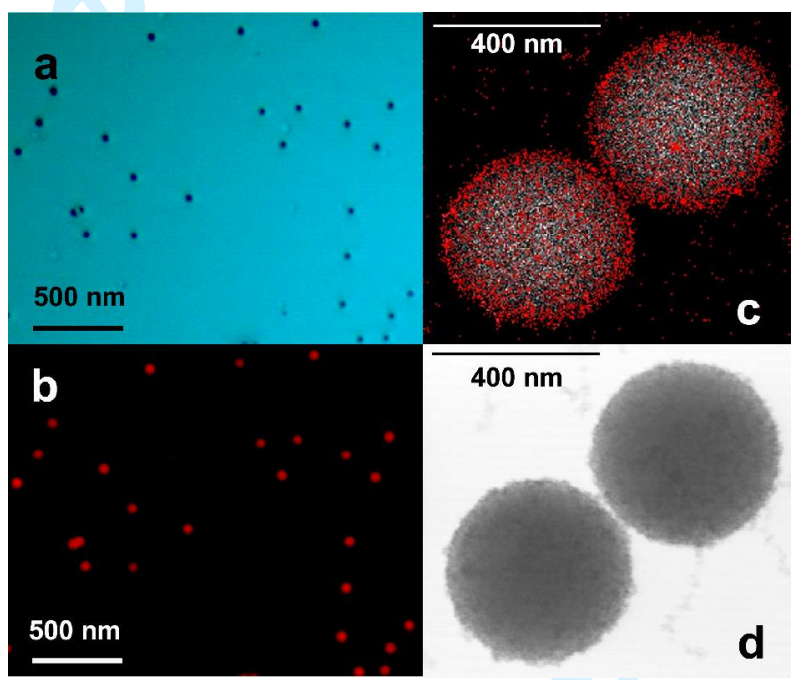

Figure 2. Images of the microspheres (a-b) from an optical microscope (a) transmission image (b) fluorescent image, (c-d) from STEM (c) distribution map of silicon (white spots) and iron (red spots) and (d) transmission image of microspheres shown in (c).

The numbers of QDs and MPs incorporated in each MS were determined by elemental analysis using inductively coupled plasma-optical emission spectroscopy (ICPOES) performed by Galbraith Laboratories, Inc. For 500-nm microspheres, QDs accounted for $1.1 \pm 0.3 \%$ of the total volume, or $2.0 \pm 1.2 \%$ of the shell volume, which corresponds to $4600 \pm 1400$ QDs per MS. The highest percent volume of MPs was $3.9 \pm 1.1 \%$ of the total volume, or $7.3 \pm 4.1 \%$ of the shell. This amount corresponds to $13000 \pm 3700$ MPs per 500nm MS (see Supporting Information for details).

The magnetic properties of these microspheres were measured using a SQUID magnetometer. Figure $3 \mathrm{a}$ shows the magnetization versus magnetic field at $5 \mathrm{~K}$ for three different microsphere samples. From the shapes of the hysteresis loops, it can be inferred that microspheres containing both MPs and QDs were ferromagnetic at $5 \mathrm{~K}$ (green and black 
lines in Figure 3a), while the microspheres with only QDs incorporated were diamagnetic (pink line). Moreover, the value of the saturation magnetization $\left(M_{S}\right)$ of each sample was measured and used to estimate the amount of $\gamma-\mathrm{Fe}_{2} \mathrm{O}_{3}$ in each sample based on the known $M_{S}$ of bulk $\gamma-\mathrm{Fe}_{2} \mathrm{O}_{3}\left(3.9 \times 10^{5} \mathrm{Am}^{-1}\right){ }^{22}$ The number of incorporated MPs per microsphere was estimated using the diameter of the microspheres and MPs measured from TEM images, the mass of the sample, and the assumption that MPs were uniformly incorporated and did not significantly alter the density of bulk $\mathrm{SiO}_{2}$. Accordingly, 500-nm microspheres with 7-nm MPs incorporated exhibited an $M_{S}$ of $5.35 \mathrm{emu} / \mathrm{g}$ and thus contained $11000 \pm 3100$ MPs per MS. This number of MPs is $20 \%$ lower than indicated by ICP-OES elemental analysis. The

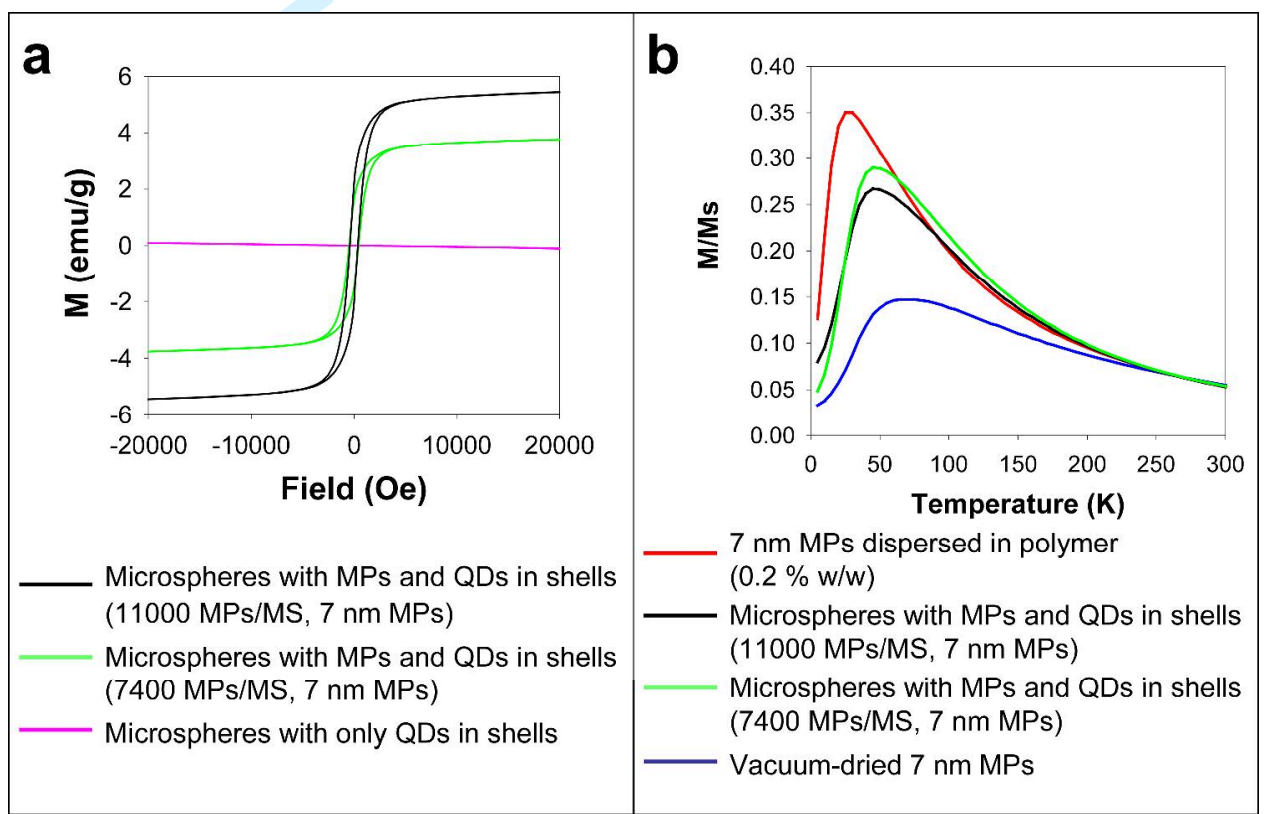

Figure 3. Magnetic characterization of the 500-nm microspheres (a) magnetization versus magnetic field at $5 \mathrm{~K}$ and (b) zero field cooled magnetization versus temperature measured in a 100 Oe field.

higher number is probably more reliable, because size ${ }^{23}$ and ligand effects ${ }^{24}$ cause $M_{S}$ of MPs to be lower than that of bulk maghemite.

In Figure $3 \mathrm{~b}, T_{B}$ corresponds to the maximum in the zero field cooled magnetization curves measured in a small field (100 Oe). As the MP concentration increases, the dipolar interaction between MPs becomes stronger, which causes $T_{B}$ to increase and the magnetization at low temperature to decrease. At high temperature, the temperaturedependent magnetization curves for samples of different concentrations converge, when thermal energy has overcome interparticle dipolar coupling. As a reference in which dipolar coupling was negligible, ${ }^{25}$ we prepared a sample of MPs dispersed in poly(laurylmethacrylate) cross-linked with ethyleneglycol dimethacrylate. ${ }^{26}$ As shown in 
Figure $3 b$, MPs dispersed in polymer have a blocking temperature of $30 \mathrm{~K}$ (red line) while the magnetic microspheres have $T_{B}$ of $45-50 \mathrm{~K}$ (green and black lines). The increase in $T_{B}$ indicates that the packing density of the MPs in the microspheres was higher than that of the MPs dispersed in polymer. ${ }^{25}$ However, when compared to vacuum-dried MPs, whose $T_{B}$ is $70 \mathrm{~K}$ (the blue line), the MPs in the microspheres had a lower $T_{B}$, which indicates that the packing density of the MPs in microspheres was still not as high as that of the dried powder MPs.

To demonstrate the combined magnetic/fluorescence characteristics of the MS, we have performed the magnetic manipulation of the microspheres in fluid. Microelectromagnets, which are lithographically-patterned conducting wires, were used to control the motion of microspheres. ${ }^{27}$ Local magnetic fields generated by microelectromagnets interact with MPs in the microspheres, which pulls the microspheres toward the maximum in the field magnitude. Two types of microelectromagnets, an array of wires and a ring trap, were prepared for this demonstration. The manipulation process can be easily monitored in real-time by observing the emission from QDs in the microspheres.

When an external magnetic field $\boldsymbol{B}$ is generated by microelectromagnets, the microspheres assume induced magnetic moments $\boldsymbol{m}=\chi V \boldsymbol{B} / \mu_{0}$, where $\chi$ and $V$ are the magnetic susceptibility and the volume of the microspheres, respectively, and $\mu_{0}$ is the permeability of vacuum. Subsequent interactions between $\boldsymbol{B}$ and $\boldsymbol{m}$ pull the microspheres towards the maximum in the magnetic field magnitude where the microspheres are trapped. The trapping potential energy of microspheres is $U=-1 / 2 \boldsymbol{m} \cdot \boldsymbol{B}=-1 / 2 \chi V B^{2} / \mu_{0}$, and microspheres remain trapped provided $|U|>k_{B} T$, where $k_{B}$ is the Boltzmann constant and $T$ is the temperature. This condition sets the criterion on the minimum magnetic field magnitude $B_{m}=\left(2 \mu_{0} k_{B} T / \chi V\right)^{1 / 2}$ required for trapping at given temperature. The microspheres used in this experiment have $\chi=0.53$ (see Supporting Information) and $V=5.9 \times 10^{7} \mathrm{~nm}^{3}$, which gives $B_{m}=6 \mathrm{G}$ at $T=300 \mathrm{~K}$. Using microelectromagnets, magnetic fields $>10 \mathrm{G}$ can be easily generated, ensuring the stable trapping of microspheres in fluids.

For straight wires, the maximum magnetic field is located around the current-carrying wire (Figure 4a). Before turning the current on, the red-emitting microspheres floated randomly over an array of wires (Figure $4 \mathrm{~b}$ ). After the third wire from the top was activated, the microspheres were immediately attracted to the third wire (Figure 4c). After the third wire was turned off, the bottom wire was switched on (Figure 4d). Additional microspheres were attracted to the bottom wire, and the cloud of microspheres shown between the third and sixth (from the top) wires, which had been trapped on the third wire, were later trapped on the 
bottom wire. This experiment demonstrated that the microspheres were responsive to small magnetic field gradients, because the microspheres which were on the third bar were moved to the bottom bar from more than $50 \mu \mathrm{m}$ away.

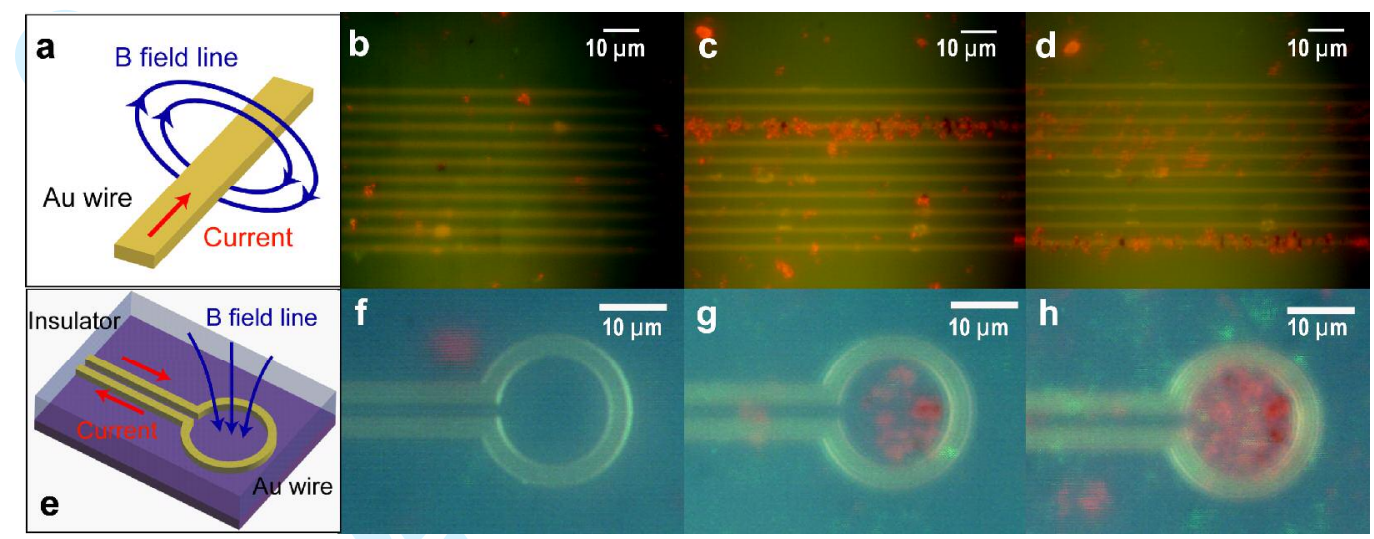

Figure 4. Images of (a-d) the straight wires trapping experiment (a) the current flow and magnetic field from the wires (b) no current in wires (c) third wire from the top turned on (d) bottom wire turned on (e-f) the ring trapping experiment (e) the current flow and magnetic field from the ring (f) no current (g) after the ring current was turned on for one minute (h) after the ring current was turned on for six minutes.

In experiments using a ring trap, we used two different types of microspheres, greenemitting microspheres without MPs incorporated, and red-emitting microspheres with MPs incorporated. (The green-emitting microspheres were in relatively low concentration, and they are difficult to discern against the background in this experiment.) In this device, the magnetic field maximum was in the middle of the ring, as shown in Figure 4e. When there was no current, both green and red microspheres floated freely over the device (Figure 4f). One minute after turning on the ring current, red-emitting microspheres were attracted to the middle of the ring (Figure 4g), more were attracted after longer on-times (Figure 4h). The green-emitting microspheres, in contrast, still floated randomly.

In conclusion, we have developed a new type of silica microsphere with tight size distribution that has both magnetic and fluorescent properties by incorporating MPs and QDs into the silica shells of the pre-made microspheres. We have also demonstrated the bifunctionality of the microspheres by manipulating the microspheres using external magnetic fields with real-time fluorescence monitoring. These microspheres have potential for biomedical applications as a probe that responds to magnetic field gradients and simultaneously luminesces. 


\section{Methods}

\section{Characterization}

Fluorescent images of the microspheres were obtained using a Nikon Eclipse ME600 epifluorescence optical microscope equipped with a Nikon DXM1200 digital camera. In order to obtain a monolayer of microspheres for light microscope imaging, we prepared samples by spin coating the microspheres dispersed in ethanol onto glass microscope slides. TEM images of the microspheres were obtained with a JEOL 200CX electron microscope operated at $200 \mathrm{kV}$. SEM images were obtained using a FEI/Philips XL30 field-emission gun-environmental scanning electron microscope (FEG-ESEM) at an acceleration voltage of $20 \mathrm{kV}$. Elemental mapping and line-scanning were done using a VG HB603 scanning transmission electron microscope operating at $250 \mathrm{kV}$ equipped with a Link Systems energydispersive X-ray analyzer. Magnetization of microspheres was studied with a SQUID (Quantum Design MPMS-5S).

\section{Preparation of MP and QD stock solutions in ethanol}

$\mathrm{CdSe} / \mathrm{ZnS}$ and $\mathrm{CdSe} / \mathrm{CdZnS}$ core/shell QDs were prepared in a two-step synthesis, similar to our previous work. ${ }^{28} \gamma-\mathrm{Fe}_{2} \mathrm{O}_{3}$ or maghemite magnetic nanoparticles (MPs) were prepared using a method modified from the literature. ${ }^{29,30}$ As an example, for synthesizing of 7-nm MPs, $400 \mu \mathrm{L}$ of $\mathrm{Fe}(\mathrm{CO})_{5}$ were added to $2 \mathrm{~mL}$ of oleic acid in $20 \mathrm{~mL}$ of dioctyl ether at $100{ }^{\circ} \mathrm{C}$. The temperature was increased at a rate of $2{ }^{\circ} \mathrm{C}$ per minute to a final temperature of $275{ }^{\circ} \mathrm{C}$, at which it was held constant for 1.5 hours. After cooling to room temperature, 0.30 $\mathrm{g}$ of $\left(\mathrm{CH}_{3}\right)_{3} \mathrm{NO}$ was added as an oxidizing agent. The mixture was heated to $130{ }^{\circ} \mathrm{C}$ for two hours, and was then heated to $275^{\circ} \mathrm{C}$ for 15 minutes. After cooling, the MPs were processed for storage by first adding ethanol to precipitate them. After centrifuging, the supernatant was discarded and the MPs were then redispersed and kept in hexanes. The MP particle size was varied by varying the molar ratio of $\mathrm{Fe}(\mathrm{CO})_{5}$ to oleic acid and the duration of heating at 275 ${ }^{\circ} \mathrm{C}$ prior to addition of $\left(\mathrm{CH}_{3}\right)_{3} \mathrm{NO}$.

The QD stock solution in ethanol was prepared using a previously reported technique. $^{15}$ In a typical procedure, as-synthesized QDs were precipitated twice with a methanol/butanol mixture to remove their native trioctylphosphine oxide (TOPO) caps, and were dried under vacuum. Next, $26 \mathrm{mg}$ of dried QDs were mixed with $195 \mathrm{mg}$ of anhydrous ethanol, $29 \mathrm{mg}$ of 3-aminopropyltrimethoxysilane (APS), and $54 \mathrm{mg}$ of 5-amino-1-pentanol 
(AP). The mixture was then heated to $40{ }^{\circ} \mathrm{C}$ for about $1 \mathrm{~h}$, leading to the formation of a clear solution of QDs.

The MP stock solution in ethanol was prepared by two slightly different procedures. The first procedure was the same as the preparation of QD solution mentioned above, with three times more AP added. The second procedure, more delicate but yielding higher MP content, the MP stock solution in ethanol was prepared using addition of 12hydroxydodecanoic acid, sonication, and syringe filtration. For example, the MPs kept in hexanes were precipitated using ethanol to remove their native oleic acid caps, and were then dried under vacuum. Then, $47 \mathrm{mg}$ of dried MPs, $95 \mathrm{mg}$ of 12-hydroxydodecanoic acid, and $1.49 \mathrm{~g}$ of ethanol were sonicated for $1 \mathrm{~h}$, yielding a clear solution of MPs. $418 \mathrm{mg}$ of AP and $772 \mathrm{mg}$ of APS were then added to the MP solution, and the mixture was heated to $40{ }^{\circ} \mathrm{C}$ for $1 \mathrm{~h}$ to ensure cap exchange with AP and APS. The mixture was then filtered through a 20$\mu \mathrm{m}$ syringe filter, leading to a clear solution of MPs.

\section{Incorporation of QDs and MPs into silica microspheres}

The procedure for incorporating MPs and QDs together into silica microspheres was adapted from Chan et al. ${ }^{15}$ In a typical procedure, $30 \mathrm{mg}$ bare silica microspheres and $16 \mathrm{mg}$ of hydroxypropyl cellulose (HPC) were added to $10 \mathrm{~mL}$ of ethanol, and this mixture was sonicated for ten minutes. 50-500 $\mu \mathrm{L}$ of MP solution and $10 \mu \mathrm{L}$ of QD solution were added into the reaction mixture while vigorously stirring, followed by the addition of $50 \mu \mathrm{L}$ of $\mathrm{H}_{2} \mathrm{O}$, $50 \mu \mathrm{L}$ of $\mathrm{NH}_{4} \mathrm{OH}$, and $0.15 \mathrm{~mL}$ TEOS. The mixture was stirred in an oil bath at $75{ }^{\circ} \mathrm{C}$ for four hours. The silica(core)/silica-MPs-QDs(shell) microspheres were then purified by performing five cycles of centrifuging, discarding the supernatant, and redispersing the microspheres in ethanol. The reaction scheme for this process is shown in Figure 1a.

\section{Demonstration of trapping of the microspheres with external magnetic fields}

The manipulations of the microspheres using external magnetic fields were performed using microelectromagnets. The fabrication process of the microelectromagnets is previously reported. ${ }^{31}$ Two types of devices used in this work were arrays of Au wires (Figure 4a) and an $\mathrm{Au}$ wire patterned into a ring (Figure 4e). The maximum current used in these experiment was $0.09 \mathrm{~A}$, corresponding to magnetic field of $\sim 67 G$ in the Au straight wire trap, and $\sim 42 G$ in the middle of the ring trap. In these trapping experiments, we used microspheres dispersed in water instead of ethanol to avoid rapid evaporation of the solvent. The microspheres were 
transferred from the ethanol medium to a water medium by two cycles of centrifugation and redispersion into water.

\section{Acknowledgment}

We thank A. J. Garratt-Reed for the scanning transmission electron microscopy work, A. Dorn for scanning electron microscopy assistance, Y. Chan, D. C. Oertel, G. P. Nair, and P. T. Snee for helpful discussions, as well as the Anandamahidol Foundation (Thailand) for a graduate fellowship awarded to N. I. This work was funded in part through the National Science Foundation Materials Research Science and Engineering Center (NSF MRSEC) program at MIT, the NSF Collaborative Research in Chemistry (NSF CRC) program, and the NSF Nanoscale Science and Engineering Center (NSF NSEC program) at Harvard University.

\section{References}

1. Pankhurst, Q. A.; Connolly, J.; Jones, S. K.; Dobson, J., Applications of magnetic nanoparticles in biomedicine. J. Phys. D: Appl. Phys. 2003, 36, R167-R181.

2. Zhu, Y. H.; Da, H.; Yang, X. L.; Hu, Y., Preparation and characterization of core-shell monodispersed magnetic silica microspheres. Colloids Surf., A 2003, 231, 123-129.

3. Yang, C. L.; Guan, Y. P.; Xing, J. M.; Liu, J. G.; Shan, G. B.; An, Z. T.; Liu, H. Z., Preparation of magnetic polystyrene microspheres with a narrow size distribution. AIChE J. 2005, 51, 2011-2015.

4. Tanyolac, D.; Ozdural, A. R., Preparation of low-cost magnetic nitrocellulose microbeads. React. Funct. Polym. 2000, 45, 235-242.

5. Muller-Schulte, D.; Schmitz-Rode, T.; Borm, P., Ultra-fast synthesis of magnetic and luminescent silica beads for versatile bioanalytical applications. J. Magn. Magn. Mater. 2005, 293, 135-143.

6. Yi, D. K.; Selvan, S. T.; Lee, S. S.; Papaefthymiou, G. C.; Kundaliya, D.; Ying, J. Y., Silica-coated nanocomposites of magnetic nanoparticles and quantum dots. J. Am. Chem. Soc. 2005, 127, 4990-4991.

7. Claesson, E. M.; Philipse, A. P., Monodisperse magnetizable composite silica spheres with tunable dipolar interactions. Langmuir 2005, 21, 9412-9419.

8. Zhang, M. J.; Itoh, T.; Abe, M., Ultrasonic visualization of still and flowing waters using contrast agents of magnetite-encapsulated porous silica microspheres. Jpn. J. Appl. Phys.,

Part 1 1997, 36, 243-246. 
9. Tartaj, P.; Gonzalez-Carreno, T.; Bomati-Miguel, O.; Serna, C. J.; Bonville, P., Magnetic behavior of superparamagnetic Fe nanocrystals confined inside submicron-sized spherical silica particles. Phys. Rev. B 2004, 69, 094401.

10. Ramesh, S.; Prozorov, R.; Gedanken, A., Ultrasound driven deposition and reactivity of nanophasic amorphous iron clusters with surface silanols of submicrospherical silica. Chem. Mater. 1997, 9, 2996-3004.

11. Krishnan, K. M.; Pakhomov, A. B.; Bao, Y.; Blomqvist, P.; Chun, Y.; Gonzales, M.; Griffin, K.; Ji, X.; Roberts, B. K., Nanomagnetism and spin electronics: materials, microstructure and novel properties. J. Mater. Sci. 2006, 41, 793-815.

12. Gupta, A. K.; Gupta, M., Synthesis and surface engineering of iron oxide nanoparticles for biomedical applications. Biomaterials 2005, 26, (18), 3995-4021.

13. Yoon, T. J.; Kim, J. S.; Kim, B. G.; Yu, K. N.; Cho, M. H.; Lee, J. K., Multifunctional nanoparticles possessing a "magnetic motor effect" for drug or gene delivery. Angew. Chem. Int. Ed. 2005, 44, 1068-1071.

14. Qiu, G. M.; Xu, Y. Y.; Zhu, B. K.; Oiu, G. L., Novel, fluorescent, magnetic, polysaccharide-based microsphere for orientation, tracing, and anticoagulation: Preparation and characterization. Biomacromolecules 2005, 6, 1041-1047.

15. Chan, Y.; Zimmer, J. P.; Stroh, M.; Steckel, J. S.; Jain, R. K.; Bawendi, M. G., Incorporation of luminescent nanocrystals into monodisperse core-shell silica microspheres. Adv. Mater. 2004, 16, 2092-2097.

16. Hong, X.; Li, J.; Wang, M. J.; Xu, J. J.; Guo, W.; Li, J. H.; Bai, Y. B.; Li, T. J., Fabrication of magnetic luminescent nanocomposites by a layer-by-layer self-assembly approach. Chem. Mater. 2004, 16, 4022-4027.

17. Fu, A. H.; Gu, W. W.; Larabell, C.; Alivisatos, A. P., Semiconductor nanocrystals for biological imaging. Curr. Opin. Neurobiol. 2005, 15, 568-575.

18. Kim, J.; Lee, J. E.; Lee, J.; Yu, J. H.; Kim, B. C.; An, K.; Hwang, Y.; Shin, C. H.; Park, J. G.; Hyeon, T., Magnetic fluorescent delivery vehicle using uniform mesoporous silica spheres embedded with monodisperse magnetic and semiconductor nanocrystals. J. Am. Chem. Soc. 2006, 128, 688-689.

19. Salgueirino-Maceira, V.; Correa-Duarte, M. A.; Spasova, M.; Liz-Marzan, L. M.; Farle, M., Composite silica spheres with magnetic and luminescent functionalities. Adv. Funct. Mater. 2006, 16, 509-514.

20. Gaponik, N.; Radtchenko, I. L.; Sukhorukov, G. B.; Rogach, A. L., Luminescent polymer microcapsules addressable by a magnetic field. Langmuir 2004, 20, 1449-1452. 
21. Smith, A. M.; Ruan, G.; Rhyner, M. N.; Nie, S. M., Engineering luminescent quantum dots for In vivo molecular and cellular imaging. Ann. Biomed. Eng. 2006, 34, 3-14.

22. Tepper, T.; Ross, C. A., Pulsed laser deposition of iron oxide films. J. Appl. Phys. 2002, 91, 4453-4456.

23. Berkowitz, A. E.; Schuele, W. J.; Flanders, P. J., Influence of crystallite size on the magnetic properties of acicular gamma- $\mathrm{Fe}_{2} \mathrm{O}_{3}$ particles. J. Appl. Phys. 1968, 39, 1261-1263. 24. Berkowitz, A. E.; Lahut, J. A.; Jacobs, I. S.; Levinson, L. M.; Forester, D. W., Spin Pinning at Ferrite-Organic Interfaces. Phys. Rev. Lett. 1975, 34, 594-597.

25. Frankamp, B. L.; Boal, A. K.; Tuominen, M. T.; Rotello, V. M., Direct control of the magnetic interaction between iron oxide nanoparticles through dendrimer-mediated selfassembly. J. Am. Chem. Soc. 2005, 127, 9731-9735.

26. Tracy, J. B.; Weiss, D. N.; Dinega, D. P.; Bawendi, M. G., Exchange biasing and magnetic properties of partially and fully oxidized colloidal cobalt nanoparticles. Phys. Rev. B 2005, 72, 064404.

27. Lee, C. S.; Lee, H.; Westervelt, R. M., Microelectromagnets for the control of magnetic nanoparticles. Appl. Phys. Lett. 2001, 79, 3308-3310.

28. Fisher, B. R.; Eisler, H. J.; Stott, N. E.; Bawendi, M. G., Emission intensity dependence and single-exponential behavior in single colloidal quantum dot fluorescence lifetimes. $J$. Phys. Chem. B 2004, 108, 143-148.

29. Woo, K.; Hong, J.; Choi, S.; Lee, H. W.; Ahn, J. P.; Kim, C. S.; Lee, S. W., Easy synthesis and magnetic properties of iron oxide nanoparticles. Chem. Mater. 2004, 16, 28142818.

30. Teng, X. W.; Yang, H., Effects of surfactants and synthetic conditions on the sizes and self-assembly of monodisperse iron oxide nanoparticles. J. Mater. Chem. 2004, 14, 774-779. 31. Lee, H.; Purdon, A. M.; Westervelt, R. M., Micromanipulation of biological systems with microelectromagnets. IEEE Trans. Magn. 2004, 40, 2991-2993. 